\title{
Design and Analyzed of Swastika-Shaped Frequency Selective Surface with Split Ring Resonator Metamaterial Absorber
}

\author{
Rahul Ateriya \\ M.E. (Microwave Engineering), Dept of ECE \\ Jabalpur Engineering College, Jabalpur, India
}

\author{
Garima Tiwari \\ Assistant Professor, dept of ECE Jabalpur \\ Engineering college, Jabalpur, India
}

\begin{abstract}
A novel metamaterial element is used as an Absorber for a Frequency range applications is presented in this paper, which shows a single negative permeability, permittivity and refractive Index characteristics at 1-10 GHz frequency ranges. The Absorber is made of coupled Swastikashaped conductors as a patch side of the dielectric substrate, which shows the characteristic of single negative properties of light at $2-2.25$ and $8.9-9.36 \mathrm{GHz}$ of negative permeability and $4-4.6,5.7-6.7$ and $6.7-9$ negative permittivity and 8.3 to $9.3 \mathrm{GHz}$ of negative refractive index. The electromagnetic wave behaves as an evanescent field, or evanescent wave at above frequencies of the proposed structure, the oscillation of electric or magnetic field that does not occurred as an electromagnetic wave at different frequencies, when it is propagate into the proposed structure, whose energy is decaying with respect to time and then the completed becomes zero. In the $8.93-9.3 \mathrm{GHz}$, the proposed structure behaves like a double negative metamaterial, which has many properties of light. The electromagnetic (EM) Wave ports is used here to analysis of Swastik-shaped structure is carried out using the full-wave Simulation method based on CST software package and then validated with the experimental results, which shows the characteristics of Absorber of the electromagnetic waves of proposed structure. To obtain $S$ parameters of the proposed structure by the use of post processing in CST, the Refractive index and permeability curves are calculated. Perfect electric and perfect magnetic (PE-PM) boundary conditions are used here in CST to extract the $S$ parameters. To excite the proposed structure, wave ports are used.
\end{abstract}

Index Terms - Metamaterial, FSS, Evanescent field, Swastikashaped metamaterial, CST, DNG (double negative) metamaterials.

\section{INTRODUCTION}

A metamaterial is not a natural material, which occurred in nature, its meaning beyond the properties of material, which not occurred naturally. This material is designed by engineers in experimental lab in our earth to have a property that is not found in naturally occurring materials. These materials are made from multiple numbers of metal unit cells from composite materials such as metals and plastics. These materials are arranged in symmetric matter at scales that are smaller than the wavelengths of the phenomena. Metamaterials derive their properties not from the properties of the base materials. their precise shape, geometry, size, and arrangement gives them their smart properties capable of manipulating electromagnetic waves by blocking, absorbing, enhancing, or bending waves, to achieve benefits that go beyond what is possible with conventional materials[1]. In electromagnetic wave an evanescent field or evanescent wave, is an oscillating electric or magnetic field that does not propagate as an electromagnetic wave but whose energy is spatially concentrated in the vicinity of the source [2]. In recent years due to the novel properties of the metamaterial, many researchers are interested in it. Metamaterials have been the most innovative concept in electromagnetic and materials fields [3]. Generally; metamaterial is periodic or non-periodic composite materials. The electromagnetic properties of metamaterial depend on material composition as well as on the inclusion of macroscopic structures which are specially designed to obtain a specific response. By introducing different structures within it, the properties of a material can be modified. Due to this behavior of metamaterials, it can be used in a wide frequency ranges, ranging from microwaves to optics frequency ranges. For improving the performance of microwave devices metamaterials are widely used. Metamaterials are employed in filters, power dividers and amplifier [4]. Apart from that, metamaterials are widely used in antenna miniaturization [5] and also modify the characteristics of antennas [6]. S-parameterretrieval method [7], are the two methods which are used to evaluate the electromagnetic properties such as Complex permeability $(\mu)$, the permittivity $(\varepsilon)$ and refractive index (n). S-parameters extracted from the actual structure and hence provide more accurate values for the permittivity, permeability, and refractive index. In this paper a new split resonator of $\mathrm{Z}$ shape has been presented. We use CST High-Frequency Structure Simulator which uses the FiniteDifference Time-Domain (FDTD) method for the extraction of S parameter.

Permeability and refractive index curves have been used as a result of evanescent wave property. In view of this, a novel Swastika-shaped metamaterial structure (Fig. 1) is proposed in this paper implying the same principle of tightly-coupled resonators [8], which show $\mu$ - negative and $\varepsilon$-negative characteristics at distinct microwave frequency ranges. The EM analysis of proposed metamaterial structure is performed using full-wave method [9] based CST Microwave Studio and then validated with the experimental results for proof-of-the concept, which exhibits excellent EM performance in terms of power transmission efficiency and bandwidth. 


\section{DESIGN OF SWASTIKA-SHAPED METAMATERIAL STRUCTURE}

The metamaterial structure proposed in this paper consists of Swastika-shaped structures, which are used as a patch which is made up of a copper conductor on either side of the substrate shown in Fig. 1. The structure is made of an Arlon AD 350 lossy material as a dielectric slab with the height of $1.8 \mathrm{~mm}$, which can be changed according to the requirement of bandwidth. To design the proposed structure, we used symmetry structure of swastika-shaped to get more appropriate simulation of the metamaterial structure. We designed the structure with $8.6 \mathrm{~mm}$ distance of length and width of the swastika shaped metamaterial.

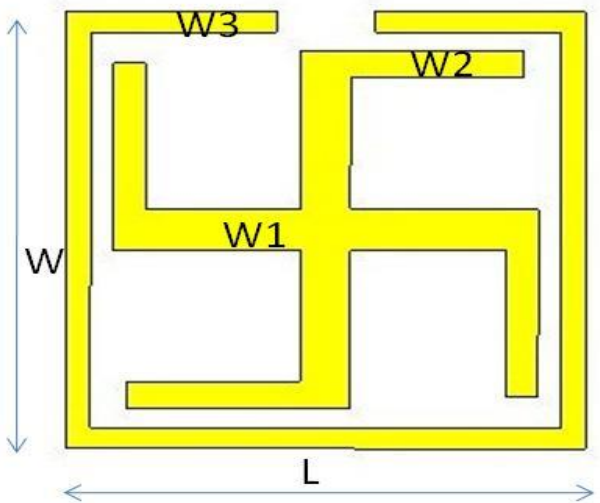

Fig.1. Schematic Diagram of Swastika-shaped FSS unit cell

The wave port is used to excite the EM wave inside the proposed structure, which can be used to analyze the characteristics of wave of light. The swastika-shaped FSS metamaterial structure with applied wave port is shown in Fig. no.2. The electromagnetic (EM) Wave ports is used here to analysis of Swastik-shaped structure is carried out using the full-wave Simulation method based on CST software package and then validated with the experimental results, which shows the characteristics of Absorber of the electromagnetic waves of proposed structure. To obtain $\mathrm{S}$ parameters of the proposed structure by the use of post processing in CST, the Refractive index and permeability curves are calculated. Perfect electric and perfect magnetic (PE-PM) boundary conditions are used here in CST to extract the $\mathrm{S}$ parameters. To excite the proposed structure, wave ports are used.

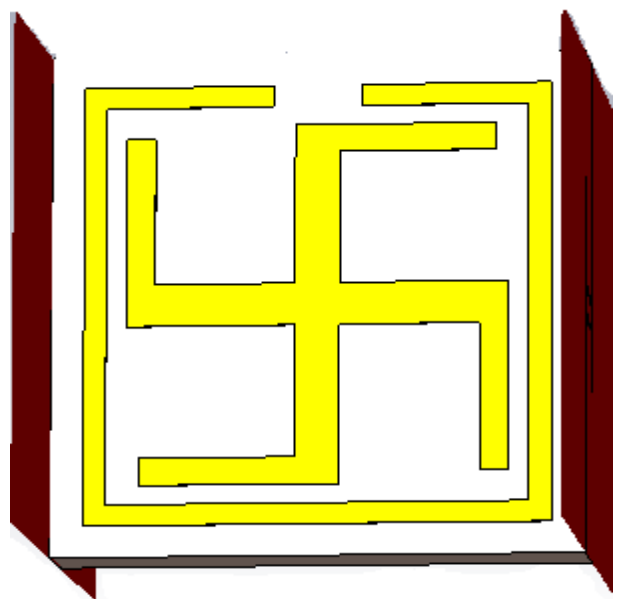

Fig.2. Schematic Diagram of Swastika-shaped FSS unit cell material with applied wave ports
The proposed structure is designed on Arlon AD 350 (lossy) substrate with relative permittivity $\varepsilon r=4.3$, a loss tangent 0.002 and a thickness of $1.8 \mathrm{~mm}$. The conducting layer is of copper with a thickness of $0.0175 \mathrm{~mm}$. The design parameters of the Swastika-shaped metamaterial unit cell are given in Table I.

Table no. I - Description of parameters of Swastikashaped FSS metamaterial

\begin{tabular}{|c|c|c|c|}
\hline S.N. & parameters & $\begin{array}{c}\text { Values } \\
(\mathrm{mm})\end{array}$ & Description \\
\hline 1 & $\mathrm{~L}$ & 8.6 & length \\
\hline 2 & $\mathrm{~W}$ & 8.6 & width \\
\hline 3 & $\mathrm{Ts}$ & 1.8 & Thickness of substrate \\
\hline 4 & $\mathrm{Tc}$ & 0.017 & Thickness of copper \\
\hline 5 & $\mathrm{~W} 1$ & 1 & $\begin{array}{c}\text { width of connected } \\
\text { lines }\end{array}$ \\
\hline 6 & W2 & 0.65 & Width of plus \\
\hline 7 & W3 & 0.50 & Width of SRR \\
\hline
\end{tabular}

III. NUMERICAL FORMULATION AND

EVUALATION OF SWASTIKA-SHAPED FSS METAMATERIAL

A novel Swastika-shaped metamaterial structure has been presented in this paper, which shows $\mu$-negative and $\varepsilon$ negative characteristics in different frequency ranges. It has been demonstrated that the metamaterial properties of bilayered Swastika-shaped structure depend on its electric (symmetric distribution) and magnetic (asymmetric distribution) resonances. This concept has been verified by numerical simulations and demonstrated experimentally. In electromagnetic, the material's characteristics are defined by the permittivity, permeability, and Conductivity. The propagation profile of the material at a different frequency is defined by the extraction of permittivity, permeability at that frequency.

To extract the permittivity and permeability of the material the refractive index and impedance of the material are used. To extract permittivity, permeability, consider the unit element of a metamaterial with lattice vectors in all three dimensions. For the simulation of the periodic metamaterial and excitation of this metamaterial in order to extract the $S$ parameters, appropriate boundary conditions and excitations are assigned to the different surface of the three-dimensional unit element. The relationship between the permittivity $(\varepsilon)$, permeability $(\mu)$ and refractive index is shown by the following expressions.

$$
\begin{aligned}
& S_{11}=\frac{R_{01}\left(1-e^{i 2 n k_{0} d}\right)}{1-R_{01}^{2} e^{i 2 n k_{0} d}} \\
& S_{21}=\frac{\left.\left(1-R_{01}^{2}\right) e^{i 2 n k_{0} d}\right)}{1-R_{01}^{2} e^{i 2 n k_{0} d}} \\
& \mu=n z \\
& \mu_{r} \sim \frac{\left(1-V_{2}\right)}{\left(1+V_{2}\right)} \frac{2}{j k_{0} d}
\end{aligned}
$$


$\varepsilon_{r}=\left(\frac{k}{k_{0}}\right)^{2} \frac{1}{\mu_{r}}$ 5

$n=\frac{k}{k_{0}}=\sqrt{\varepsilon_{r} \mu_{r}}$ 6

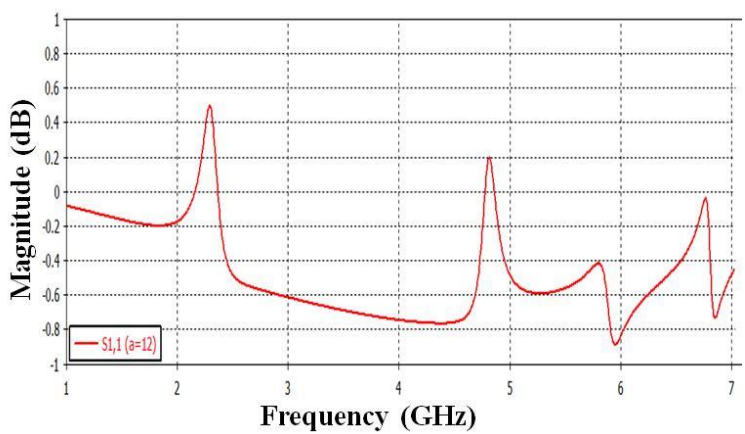

Fig.3. Reflection coefficient (S11) of Swastika-shaped FSS

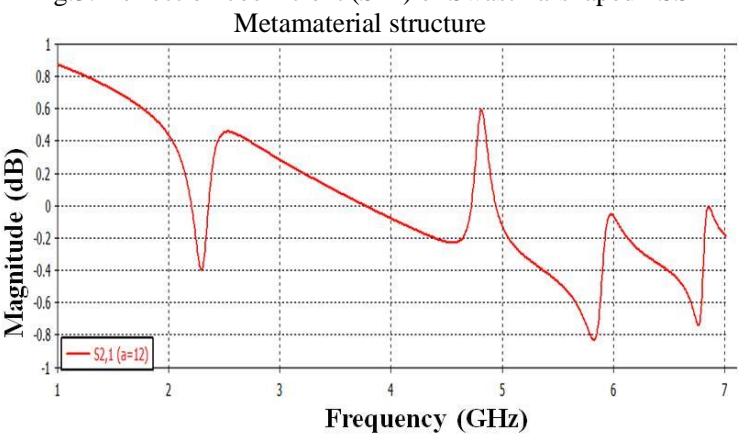

Fig.4. Transmission coefficient (S21) of Swastika-shaped FSS Metamaterial structure

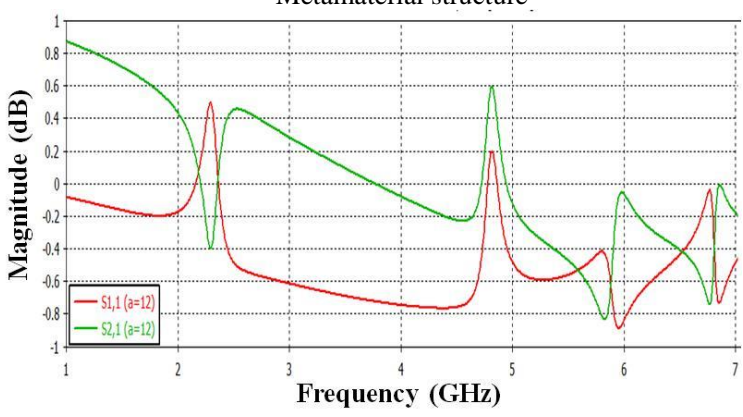

Fig.5. S-Parameters of Swastika-shaped FSS Metamateria

\section{RESULTS OF SWASTIKA-SHAPED FSS METAMATERIAL}

To get better results within the desirable range of frequency, we use CST software for simulation to get S parameters of the proposed structure. For further, S parameters use as a primary data for the simulation of permeability, permittivity and refractive index of the swastika-shaped metamaterials unit cell. The permeability properties of the metamaterial unit cell are shown in Fig. no.6, the permittivity of the metamaterial unit cell is shown in fin. 7 and the refractive index are shown in Fig. no.8. The permeability of the proposed structure works as single negative properties of light, the electromagnetic wave decaying at above frequency ranges with respect to time the oscillation of Electromagnetic wave becomes zero inside the structure, which is behaves at different frequencies ranges as an Absorber with $1-10 \mathrm{GHz}$ frequency ranges. The double negative metamaterial of the proposed structure is shown in fig. no. 9, which showing double negative properties of light and has many applications of light.

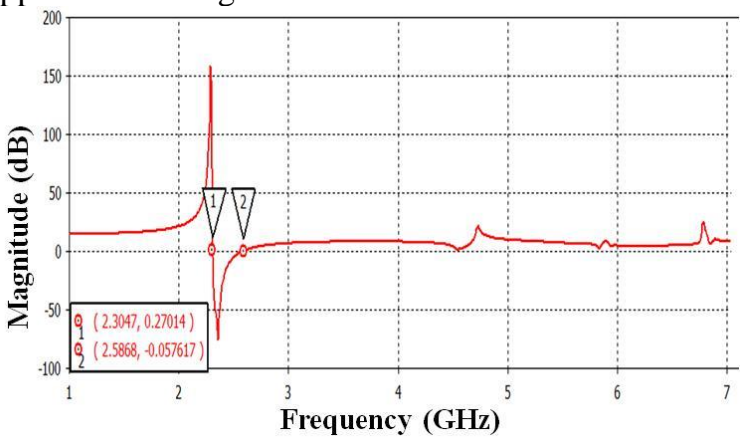

Fig.6. Permeability of swastika-shaped FSS Metamaterial

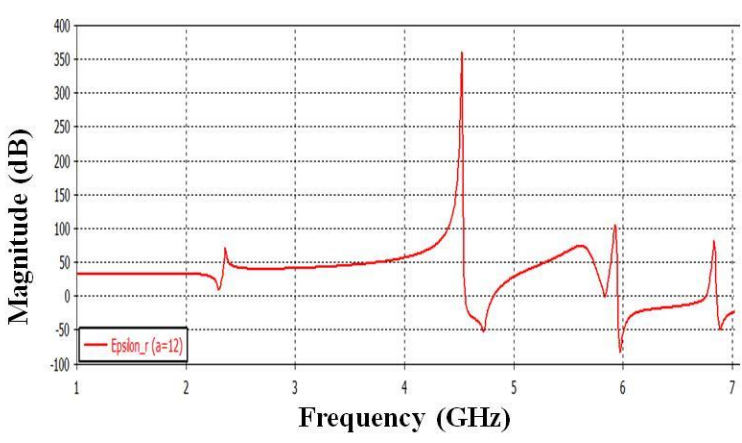

Fig.7. Permittivity of swastika-shaped FSS Metamaterial

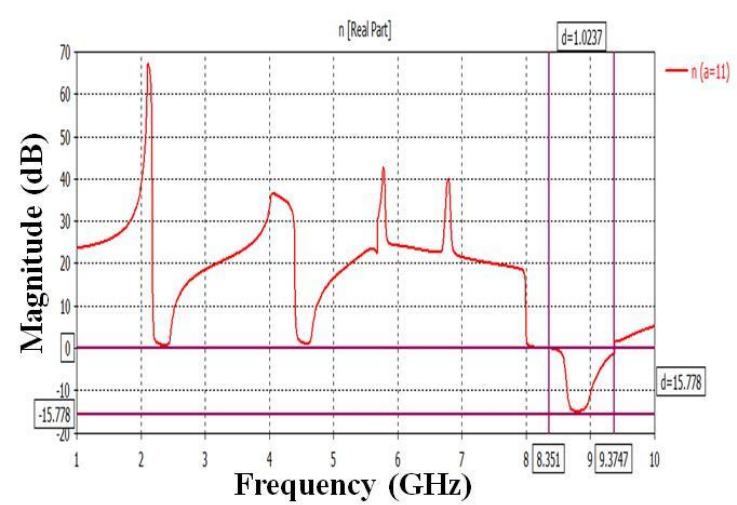

Fig.8. Refractive Index of swastika-shaped FSS Metamaterial

The electromagnetic wave behaves as an evanescent field, or evanescent wave in fig. no.6 and fig.no.7 of the proposed structure, the oscillation of electric or magnetic field that does not occurred as an electromagnetic wave at different frequencies.

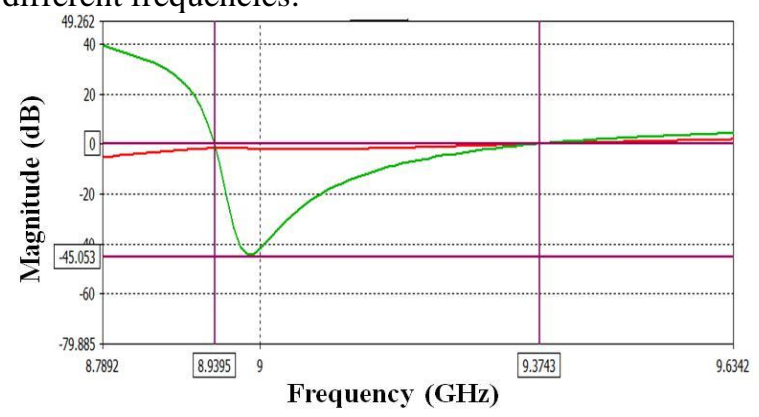

Fig.9. Double negative field of swastika-shaped FSS Metamaterial 
The description of permeability, permittivity and refractive index of the Swastika-shaped frequency selective surface metamaterial unit cell is shown in Table no II.

\section{TABLE NO.II - DISCRIPTION OF PREMEABILITY AND REFRACTIVE INDEX OF SWASTIK-SHAPED FSS METAMATERIAL}

\begin{tabular}{|c|c|c|c|}
\hline S.N. & PARAMETERS & $\begin{array}{c}\text { VALUE } \\
(\mathbf{G H z})\end{array}$ & $\begin{array}{c}\text { RANGE } \\
(\mathbf{G H z})\end{array}$ \\
\hline 1. & PERMEABILITY & -70 & $2-2.5$ \\
& & -40 & $8.9-9.36$ \\
\hline 2. & PERMITTIVITY & -112 & $4-4.6$ \\
& & -100 & $5.7-6.7$ \\
& & -58 & $6.7-9$ \\
\hline 3 & REFRACTIVE INDEX & -15.036 & $8.3-9.3$ \\
\hline 4 & DNG & -45 & $8.9-9.3$ \\
\hline
\end{tabular}

\section{CONCLUSION}

A Swastika-shaped Frequency selective surface metamaterial is designed and analyzed for used as a absorber in 1-10 GHz frequency range and it can be used as a single negative materials, these characteristics of light the proposed structure is useful in this frequency range, which shows the characteristic of single negative properties of light at $2-2.25$ and $8.9-9.36 \mathrm{GHz}$ of negative permeability and $4-4.6,5.7-6.7$ and $6.7-9$ negative permittivity and 8.3 to $9.3 \mathrm{GHz}$ of negative refractive index. . The electromagnetic wave behaves as an evanescent field, or evanescent wave at above frequencies ranges of the proposed structure, the oscillation of electric or magnetic field that does not occurred as an electromagnetic wave at above frequencies ranges, when it is propagate into the proposed structure, whose energy is decaying with respect to time and then the completed becomes zero. In the $8.93-9.3 \mathrm{GHz}$, the proposed structure behaves like a double negative metamaterial, which has many properties of light. For the extraction of $\mathrm{S}$ parameter using CST software is more accurate simulation of EM wave inside the boundary condition, which can be used further, simulation of permeability and refractive index of the proposed structure, which shows the characteristics of evanescent wave of light in $1-10 \mathrm{GHz}$ frequency range. At different frequencies ranges, the proposed structure can be used as good Absorber of the 1$10 \mathrm{GHz}$ frequency ranges. Perfect electric and perfect magnetic (PE-PM) boundary conditions applied to get better simulation of the proposed structure in this frequency ranges.

\section{REFERENCES}

[1] https://en.wikipedia.org/wiki/Metamaterial.

[2] https://en.wikipedia.org/wiki/Evanescent_field.

[3] Ali Khoshniat and Ramesh Abhari, Senior Member, IEEE, "Design and Evaluation of Radiated Emission Metamaterial Absorbers", June 13, 2019.

[4] Shiv Narayan, Gitansh Gulati, Sangeetha B., and Ravendra nath U. Nair, "Novel metamaterial-element based FSS for airborne redome applications", IEEE, 2018.

[5] Komsan Kanjanasit and Changhai Wang, Member, IEEE," A Wideband Resonant Cavity Antenna Assembled Using a Micro-machined CPW Fed Patch Source and a Two-Layer Metamaterial Superstrate", 2018.

[6] H. B. Wang and Y. J. Cheng, "Frequency selective surface with miniaturized elements based on quarter-mode substrate integrated waveguide cavity with two poles," IEEE, Mar. 2016.

[7] T. Haq, M. F. Khan, and O. F. Siddiqui, "Design and implementation of waveguide band-pass filter using complementary meta-resonator" IEEE, 2016.

[8] N. A. Estep and A. N. Askarpour and A. Al, "Experimental demonstration of negative-index propagation in a rectangular waveguide loaded with complementary split-ring resonators," IEEE, 2016.

[9] Y. Liu, Y. Hao, H. Wang, K. Li, and S. Gong, "Low RCS micro-strip patch antenna using frequency-selective surface and micro-strip resonator," IEEE, 2015. 